\section{Pré-natal em mulheres usuárias do Sistema Único de Saúde em duas maternidades no Estado do Rio de Janeiro, Brasil: a cor importa?}

\section{Prenatal care in women using the Brazilian National Health System (SUS) in two maternity hospitals in the State of Rio de Janeiro, Brazil: does color matter?}

Abstract

Objectives: to identify factors associated with inadequate prenatal care, with a special focus on skin color, among female users of the SUS in the State of Rio de Janeiro (RJ), in the final trimester of 2011.

Methods: a cross-sectional cohort study was carried out at two public maternity hospitals in RJ. Interviews were conducted with 1790 women bearing children, in order of sequence. The number of consults and the date on which accompaniment of the women commenced were gathered from prenatal cards. The outcome was adequate prenatal care, classified according to the Adequate Use of Prenatal Care Index. The main factor associated with exposure was skin color, along with other socio-demographic and reproductive co-variables. Multivariate logistic regression used hierarchical levels: the variables predisposing women to the use of prenatal care were on the first and second levels, respectively.

Results: dark skin color remained associated with inadequate prenatal care, even after adjustment for co-variables (OR=1.37; CI95\%:1.02-1.83). Teenage pregnancy $(O R=1.85 ; C 195 \%: 1.43-2.41)$; absence of a partner $(O R=1.75 ; C I 95 \%: 1.38-2.20)$ and multiparity (OR=2.40; CI95\%:1.49-3.85) were also positively associated with the outcome. Prenatal care in public services (OR=0.67; CI95\%: 0.49-0.91) and primiparity $(O R=0.41 ; C 195 \%: 0.32$ and 0.52$)$ were protective factors.

Conclusions: pregnant women who were black stood a greater chance of receiving inadequate prenatal care in RJ. Policies are needed to reduce inequalities relating to color/race in the prenatal care of this population.

Key words Prenatal care, Health inequalities, Social inequities, Quality of health care, Brazil
Sandra Costa Fonseca 1

Pauline Lorena Kale 2

Katia Silveira da Silva 3
1 Instituto de Saúde da Comunidade. Universidade Federal Fluminense. Rua Marquês do Paraná, 303. Prédio Anexo, $3^{\circ}$ andar. Niterói, RJ, Brasil. E-mail: sandracfonseca@yahoo.com.br

2 Instituto de Estudos em Saúde Coletiva. Universidade Federal do Rio de Janeiro. Rio de Janeiro, RJ, Brasil.

3 Instituto Fernandes Figueira. Rio de Janeiro, RJ, Brasil.

\section{Resumo}

Objetivos: identificar fatores associados ao prénatal inadequado, com destaque para cor da pele, em usuárias do SUS do Estado do Rio de Janeiro (RJ), no último trimestre de 2011.

Métodos: estudo de corte seccional em duas maternidades públicas no RJ. Foram entrevistadas 1790 parturientes, arroladas sequencialmente. Número de consultas e início do acompanhamento foram coletados dos cartões de pré-natal. O desfecho foi adequação do pré-natal, classificada pelo Índice de Adequação de Utilização do Pré-natal. A exposição principal foi cor da pele, além de covariáveis sociodemográficas e reprodutivas. A regressão logística multivariada usou níveis hierárquicos: variáveis predisponentes e capacitantes da utilização do pré-natal no primeiro e segundo nivel, respectivamente.

Resultados: a cor da pele preta manteve-se associada ao pré-natal inadequado, mesmo após ajuste para covariáveis (OR=1,37; IC95\%:1,02-1,83). Gravidez adolescente (OR=1,85; IC95\%:1,43-2,41); ausência de companheiro $(O R=1,75$; IC95\%:1,382,20) e multiparidade (OR=2,40; IC95\%:1,49-3,85) também se associaram positivamente ao desfecho. $O$ atendimento ao pré-natal em serviços públicos $(O R=0,67 ;$ IC95\%: 0,49-0,91) e primiparidade $(O R=0,41 ;$ IC95\%: 0,32 e 0,52) tiveram efeito protetor.

Conclusões: gestantes de cor preta tiveram maior chance de realizar pré-natal inadequado no RJ. São necessárias politicas para reduzir iniquidades de raça/cor na assistência pré-natal desta população. Palavras-chave Cuidado pré-natal, Desigualdades em saúde, Iniquidade social, Qualidade da assistência à saúde, Brasil 


\section{Introdução}

A atenção pré-natal adequada é reconhecidamente um fator protetor para desfechos perinatais e neonatais, ocasionando a redução da natimortalidade e da morbimortalidade neonatal, $1-2$ provavelmente por aumentar o rastreio e tratamento de infecções e problemas nutricionais maternos ${ }^{1}$ e diminuir o risco de baixo peso ao nascer, ${ }^{3-4}$ Além disso, o acompanhamento pré-natal é uma intervenção efetiva na prevenção da morbidade e morte materna. ${ }^{1,5-7}$

No Brasil, apesar de a cobertura pré-natal vir aumentando, ${ }^{8}$ ainda há deficiências na qualidade da assistência 9 e desigualdades regionais tanto em relação ao acesso quanto à realização dos procedimentos recomendados durante o pré-natal.8,10-16

Em relação às desigualdades raciais na atenção ao pré-natal, não há consenso entre os autores no Brasil. Estudo conduzido no município do Rio de Janeiro, Estado do Rio de Janeiro, no período de 1999 a 2001, mostrou que gestantes de cor preta ou parda tiveram maior chance de realizar pré-natal inadequado quando comparadas às brancas. 10 Estes resultados se repetiram nesse mesmo município entre 2007 e 200811 e na cidade de Niterói, no mesmo Estado, entre 2001 e 2009.12 Na cidade de São Luís, no Maranhão, foi relatado que mulheres pretas e pardas tiveram maior prevalência de prénatal inadequado, em 2010.13 Por outro lado, no Rio Grande do Sul, em Porto Alegre, no ano de 2002,14 em Pelotas, em 2004,15 e em populações pobres do Nordeste, 16 no ano de 2008, a cor da pele, mesmo após ajuste para variáveis socioeconômicas, não mostrou associação.

A revisão sistemática de Feijen-de Jong et al.17 avaliou a inadequação do pré-natal, de acordo com a utilização dos serviços, em países desenvolvidos e, para a variável cor da pele, verificou que mulheres pretas tendem a iniciar mais tardiamente e a receber um número menor de consultas de pré-natal. Contudo, esta evidência foi considerada ainda insuficiente, tanto por problemas de validade interna quanto pela variedade de definições para adequação do pré-natal.

No Brasil, as disparidades raciais têm sido relacionadas ao acesso e à utilização de serviços de saúde, assim como aos desfechos maternoinfantis. ${ }^{18-20}$ Mais estudos sobre a variável cor, independentemente de outros fatores socioeconômicos, têm sido recomendados. $18-19$

Este estudo investigou caraterísticas marcadoras de desigualdades sociais, com ênfase na cor da pele, quanto à adequação do pré-natal em uma população de usuárias dos serviços do Sistema Único de Saúde
(SUS), no Estado do Rio de Janeiro, Brasil.

\section{Métodos}

Este estudo faz parte de uma pesquisa, realizada em parceria com o Instituto de Saúde da Comunidade, da Universidade Federal Fluminense (ISC / UFF), Instituto de Estudos em Saúde Coletiva, da Universidade Federal do Rio de Janeiro (IESC / UFRJ), Instituto Fernandes Figueira (IFF / FIOCRUZ) e Faculdade de Saúde Pública, da Universidade de São Paulo (FSP / USP). O estudo realizado no Estado do Rio de Janeiro constituiu uma adaptação do projeto original elaborado por pesquisadores da FSP-USP, ${ }^{21}$ e incluiu outros dados socioeconômicos e hábitos de vida das gestantes.

O estudo, de delineamento transversal, foi conduzido de setembro a dezembro de 2011 em duas maternidades do SUS, dos municípios de Niterói e Rio de Janeiro (RJ), no Estado do Rio de Janeiro. A maternidade localizada em Niterói é referência para gravidez de risco da Região Metropolitana II e o número de nascidos vivos em 2011 foi 2800 (12\% do total de nascidos vivos desta região), representando $42,6 \%$ e $36 \%$ dos nascimentos no SUS em Niterói e São Gonçalo, respectivamente. A maternidade do RJ, localizada na Região Metropolitana I, atende, sem restrição de risco, principalmente a clientela residente na Área de Planejamento de Saúde a que pertence (divisão programática da Secretaria Municipal de Saúde e Defesa Civil do Rio) e o número de nascidos vivos em 2011 foi 6372 (7,2\% dos nascidos vivos da cidade e $11,8 \%$ quando se considera apenas o SUS).

As mulheres foram arroladas sequencialmente $\mathrm{e}$ a coleta de dados realizou-se em todos os dias da semana, por meio de entrevista à parturiente após 12 horas do parto por equipe previamente treinada. As mães de gemelares foram contabilizadas apenas uma vez (mantendo a unidade de análise parturiente).

O cálculo da amostra utilizou uma prevalência de pré-natal inadequado de $30 \%$, segundo estudos em populações semelhantes, ${ }^{11,12}$ que foi considerada para o grupo não exposto. A odds ratio estimada foi de 1,5 (39\% em pretas e $30 \%$ em brancas e pardas); uma razão de não exposto/ exposto de 3,3 (77\% pardas e brancas/ $23 \%$ pretas), um poder de $90 \%$ e um erro alfa de $5 \%$. O tamanho amostral mínimo obtido foi de 1640 puérperas.

O estudo usou uma amostra estratificada proporcional, considerando o número total de partos realizados em cada maternidade. A fórmula $(\mathrm{f}=\mathrm{n} / \mathrm{N})$ calculou a fração de amostragem e $(\mathrm{n} 1=\mathrm{f} . \mathrm{N} 1 / \mathrm{N})$ o número de elementos observados em cada hospital. 
A estratégia de coleta de dados sequencial no mesmo período contribuiu para esta proporcionalidade, pois sendo o período de coleta de dados o mesmo para as duas maternidades e as entrevistas realizadas à medida que ocorre o parto, o número de puérperas entrevistadas é proporcional ao número total de partos realizados em cada maternidade durante o período de um ano.

A variável dependente foi a adequação do prénatal, segundo o índice de adequação de utilização do pré-natal, proposto por Kotelchuck, 22 que combina o mês de início do pré-natal (recomendado até o $4^{\circ}$ mês de gestação) e o número recomendado de consultas de acordo com a idade gestacional (medido em percentual de consultas realizadas, de menos de $50 \%$ a mais de $109 \%$ ). Este índice apresenta quatro categorias: 1) mais do que adequado (início precoce e mais de $109 \%$ de consultas realizadas); 2) adequado (início precoce e $80-109 \%$ das consultas); 3 ) intermediário (início precoce e 50 $79 \%$ das consultas) e 4 ) inadequado (início tardio e/ou menos de $50 \%$ das consultas).

Neste estudo, baseado nas adaptações nacionais do índice de Kotelchuck, 14,23 o pré-natal foi definido como inadequado quando o seu início ocorreu após o $4^{\circ}$ mês de gravidez e/ou quando o número de consultas foi menor que $50 \%$ do recomendado para a idade gestacional: pelo menos duas consultas até menos de 22 semanas; três, entre 22 e 27 semanas; quatro, entre 28 e 31 semanas; cinco, entre 32 e 36 semanas; seis, entre 37 e 39 semanas, e sete, para 40 semanas ou mais. As categorias classificadas como pré-natal intermediário, adequado e mais que adequado, de acordo com o índice de Kotelchuck, foram agrupadas em uma única categoria que foi classificada como pré-natal adequado, ficando a variável dependente dicotomizada em adequado e inadequado.

A organização das variáveis independentes no modelo hierarquizado foi feita de acordo com o modelo teórico de Andersen, 24 que propõe fatores inter-relacionados, tanto individuais como contextuais, determinando o acesso e a utilização dos serviços de saúde. Os fatores predisponentes, como idade, raça, escolaridade, situação conjugal, redes sociais ou status na comunidade, em geral existentes antes do problema de saúde, determinariam a busca por serviços, mas seriam intermediados por fatores capacitantes para a utilização, como renda, segurosaúde e a própria disponibilidade e organização dos serviços. Ainda no modelo estaria a necessidade de saúde, representada pelos eventos/agravos de saúde que demandariam atendimento de maior ou menor complexidade. Este modelo tem sido aplicado em outros estudos de adequação do pré-natal.11,13,17
Neste estudo, foram consideradas variáveis predisponentes: a idade materna $(\leq 19$ anos, 20-34 anos, 35 e mais); cor da pele auto-referida (branca, preta e parda); grau de escolaridade da mãe $(<4$ anos; 4-7anos; 8-11 anos e 12 ou mais anos de estudo); paridade, segundo número de partos anteriores (nenhum, 1-3, $\geq 4$ ); situação de chefe da família (própria parturiente ou outro membro da família); viver com companheiro (sim ou não). As variáveis capacitantes foram renda mensal familiar per capita (dividida em tercis) e categoria do pré-natal (público ou privado). A variável não foi incluída por não de dispor do histórico completo da gestação das mulheres.

Inicialmente, a distribuição de frequência das variáveis foi descrita e, em seguida, as variáveis foram categorizadas quando pertinente, antes da análise da associação das variáveis independentes com o desfecho de interesse. Os fatores associados ao pré-natal inadequado foram investigados por regressão logística, em um modelo hierarquizado. No primeiro nível foram incluídas as variáveis predisponentes e no segundo nível, as capacitantes. Na primeira etapa foram realizadas análises univariadas para investigar a associação de cada fator com o desfecho. Aquelas variáveis associadas ao desfecho em um nível de significância de $p<0,20$ foram incluídas no modelo multivariado. $\mathrm{O}$ modelo final foi composto pelas variáveis que após ajuste se mantiveram associadas $(p<0,05)$.

No primeiro nível, as variáveis foram ajustadas entre si. No segundo nível, foram ajustadas entre si, incluindo as variáveis do primeiro nível, que se mantiveram independentemente associadas ao evento (inadequação do pré-natal), de acordo com o modelo de análise hierarquizada. ${ }^{25}$ Foram estimadas as razões de chances (OR) e seus respectivos intervalos de confiança (IC) de $95 \%$ para a associação do pré-natal inadequado com as variáveis independentes. Adicionalmente foram testadas interações entre a cor da pele e as variáveis renda e local do pré-natal. Os dados da pesquisa original foram armazenados e analisados no pacote estatístico SPSS ${ }^{\circledR}$, versão 17.

O projeto de pesquisa foi aprovado pelo Comitê de Ética em Pesquisa do IESC / UFRJ (parecer $n^{\circ}$ $35 / 2011$, processo $\left.n^{0} 15 / 2010\right)$. Foi solicitado o consentimento informado a todas as gestantes elegíveis ou às respectivas responsáveis quando as mesmas eram menores de 18 anos.

\section{Resultados}

Do total de 1874 parturientes elegíveis, houve 84 perdas $(4,4 \%)$, sendo 28 por alta precoce e 56 por 
recusa da mulher, totalizando 1790 participantes. Observou-se um percentual elevado de adolescentes na amostra $(26,6 \%)$ e menos de $10 \%$ de parturientes com 35 anos ou mais. Predominaram mulheres pardas e brancas $(72,7 \%)$ e com 8 a 11 anos de estudo $(66,0 \%)$. Quase um terço vivia sem o companheiro e mais de $10 \%$ eram chefes de suas famílias. A renda familiar era muito baixa, apenas $31 \%$ ultrapassaram $\mathrm{R} \$ 500,00$ como renda mensal per capita, equivalente a 280 dólares no período estudado (Tabela 1).

Pouco menos de $50 \%$ eram primíparas, e a maioria realizou pré-natal em serviços públicos. Cerca de $70 \%$ conseguiu iniciar as consultas até o quarto mês e quase a metade atingiu sete ou mais consultas (Tabela 2). Quanto à adequação do prénatal, um percentual baixo conseguiu se classificar como adequado. Destaca-se que 54 (3\%) mulheres não realizaram nenhuma consulta de pré-natal.

Na Tabela 3, apresentam-se as razões de chance brutas e ajustadas para o pré-natal inadequado. $\mathrm{Na}$ análise não ajustada, primiparidade e utilização dos serviços públicos se mostraram negativamente associados ao pré-natal inadequado, ambos com significância estatística. Os fatores associados positivamente à inadequação foram: ser adolescente, cor da pele preta, baixa escolaridade, viver sem companheiro, ser a própria chefe da família, multiparidade e renda familiar baixa.

Após o ajuste, observou-se que, no primeiro nível, mantiveram-se associados positivamente com o pré-natal inadequado: adolescência $(\mathrm{OR}=1,85$; IC95\%: 143-2,41); cor da pele preta $(\mathrm{OR}=1,37$; IC95\%: 1,02-1,83); viver sem companheiro $(\mathrm{OR}=1,75$; IC95\%: 1,38-2,20); multiparidade $(\mathrm{OR}=2,40$ e IC95\%: 1,49-3,85). Primiparidade se manteve negativamente associada e a escolaridade mudou a direção da associação, porém sem significância estatística.

No segundo nível, a faixa mais baixa de renda familiar manteve associação positiva com pré-natal inadequado, porém perdeu magnitude $(\mathrm{OR}=1,34$; IC95\%: 0,97-1,85) e ficou com significância estatística limítrofe $(p=0,06)$. As interações testadas não alcançaram significância estatística e não foram mostradas na Tabela. $\mathrm{O}$ atendimento em serviço público se manteve negativamente associado, com OR de 0,67 (IC95\%: 0,49-0,91).

Tabela 1

Características sociodemográficas das mulheres admitidas em duas maternidades públicas, RJ, Brasil, 2011.

\begin{tabular}{|c|c|c|}
\hline Variáveis & $\mathbf{N}$ & $\%$ \\
\hline \multicolumn{3}{|l|}{ Idade materna (anos) } \\
\hline$<20$ & 476 & 26,6 \\
\hline $20-34$ & 1155 & 64,5 \\
\hline$\geq 35$ & 159 & 8,9 \\
\hline \multicolumn{3}{|l|}{ Cor da pele } \\
\hline Branca & 488 & 27,3 \\
\hline Parda & 888 & 49,6 \\
\hline Preta & 414 & 23,1 \\
\hline \multicolumn{3}{|l|}{ Escolaridade (anos) } \\
\hline$<4$ & 52 & 2,9 \\
\hline $4-7$ & 490 & 27,4 \\
\hline 8-11 & 1181 & 66,0 \\
\hline$\geq 12$ & 67 & 3,7 \\
\hline \multicolumn{3}{|l|}{ Chefe da família } \\
\hline Própria mulher & 214 & 12,0 \\
\hline Outra pessoa & 1545 & 86,3 \\
\hline Ignorado & 31 & 1,7 \\
\hline \multicolumn{3}{|l|}{ Estado marital } \\
\hline Com companheiro & 1233 & 68,9 \\
\hline Sem companheiro & 526 & 29,4 \\
\hline Ignorado & 31 & 1,7 \\
\hline \multicolumn{3}{|c|}{ Renda familiar per capita (por tercis) } \\
\hline$>\mathrm{R} \$ 500,00$ & 415 & 23,2 \\
\hline $\mathrm{R} \$ 251,00$ a 500,00 & 662 & 37,0 \\
\hline$<\mathrm{R} \$ 250,00$ & 557 & 31,1 \\
\hline Ignorado & 156 & 8,7 \\
\hline
\end{tabular}


Características reprodutivas e do pré-natal das mulheres admitidas em duas maternidades públicas, RJ, Brasil, 2011.

\begin{tabular}{|c|c|c|}
\hline Variáveis & $\mathbf{N}$ & $\%$ \\
\hline \multicolumn{3}{|l|}{ Paridade } \\
\hline Nenhum filho anterior & 864 & 48,3 \\
\hline $1-3$ & 832 & 46,5 \\
\hline$\geq 4$ & 92 & 5,1 \\
\hline Ignorado & 2 & 0,1 \\
\hline \multicolumn{3}{|l|}{ Categoria do pré-natal } \\
\hline Não realizado & 54 & 3,0 \\
\hline Público & 1547 & 86,4 \\
\hline Particular & 189 & 10,6 \\
\hline \multicolumn{3}{|l|}{ Número de consultas } \\
\hline Nenhuma & 54 & 3,0 \\
\hline $1-3$ & 204 & 11,4 \\
\hline 4-6 & 637 & 35,6 \\
\hline$\geq 7$ & 889 & 49,7 \\
\hline Ignorado & 6 & 0,3 \\
\hline \multicolumn{3}{|l|}{ Mês de início do pré-natal } \\
\hline Sem pré-natal & 54 & 3,0 \\
\hline Primeiro ao quarto mês & 1230 & 68,7 \\
\hline Quinto ao sétimo & 418 & 23,4 \\
\hline Após o sétimo & 45 & 2,5 \\
\hline Ignorado & 43 & 2,4 \\
\hline \multicolumn{3}{|l|}{ Adequação do pré-natal } \\
\hline Adequado & 425 & 23,7 \\
\hline Intermediário & 744 & 41,6 \\
\hline Inadequado & 621 & 34,7 \\
\hline
\end{tabular}




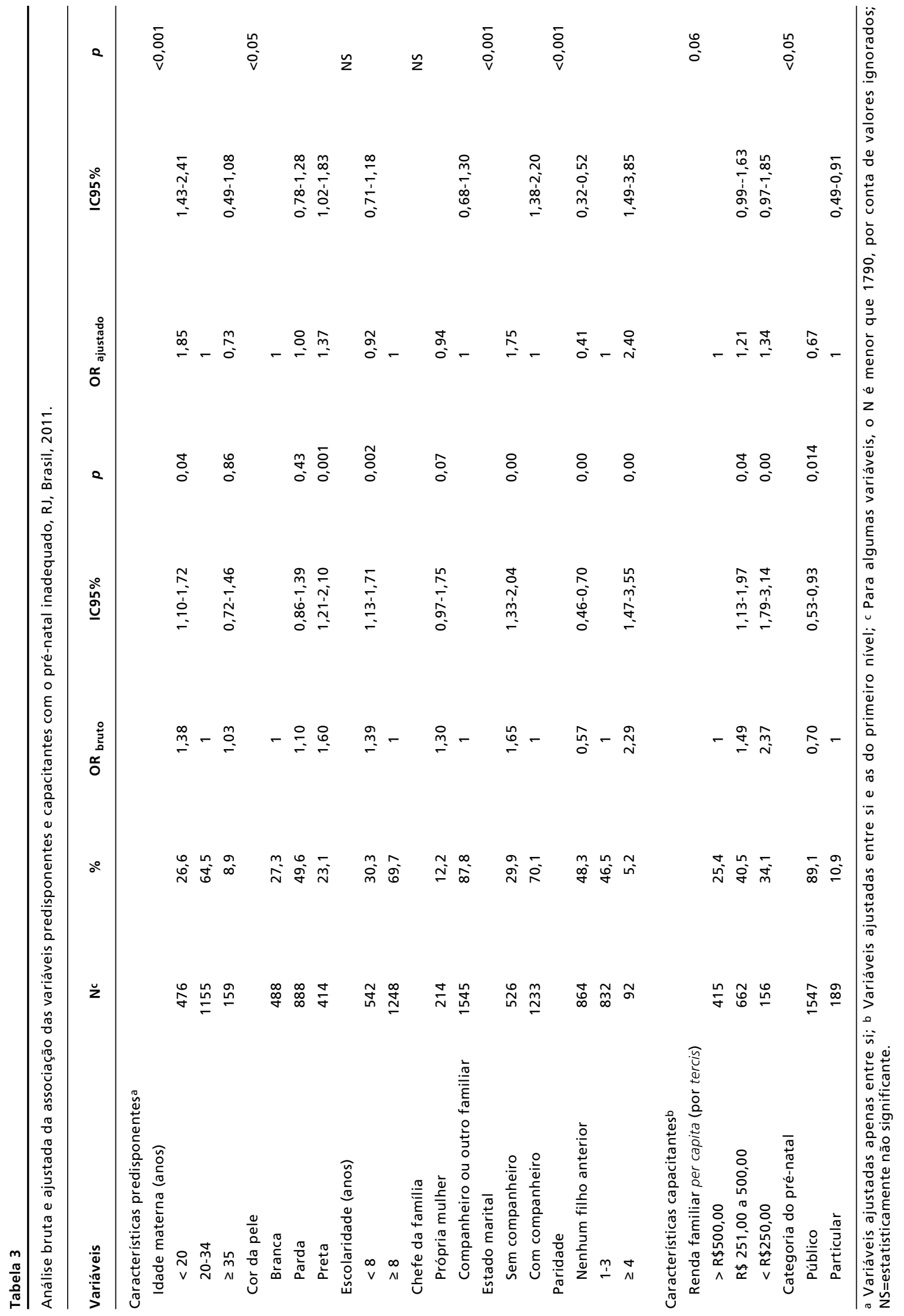




\section{Discussão}

O estudo mostrou o elevado percentual de pré-natal inadequado $(34,7 \%)$ entre as mulheres estudadas. A frequência de inadequação foi superior à apontada por Viellas et al., ${ }^{8}$ na Região Sudeste, mas esta diferença pode ser explicada pelas características da população de estudo, constituída apenas por usuárias do SUS, enquanto que o estudo de Viellas et al. ${ }^{8}$ abrangeu toda a população.

As mulheres de cor da pele preta apresentaram $37 \%$ mais chance de receber atenção inadequada no pré-natal. Outras variáveis sociodemográficas, como idade materna e ausência de companheiro, também foram associadas à inadequação.

A cor da pele, como marcador de iniquidades raciais ou étnicas, tem sido discutida na literatura e as conclusões não são consensuais. $26,27 \mathrm{Um}$ dos problemas apontados é a diferente forma de aferição da variável e sua conceituação. Em nosso estudo, optamos por usar a informação da cor auto-referida, nos moldes dos sistemas de informação nacionais, facilitando a comparabilidade com outros estudos.

Achados semelhantes em relação ao pré-natal no Brasil foram reportados no Estado do Rio10,12 e no Maranhão,13 considerando bases populacionais completas e com critérios de adequação baseados na proposta de Kotelchuck. No estudo de Domingues et al.,11 foram estudadas gestantes durante consultas no pré-natal da rede SUS na cidade do Rio de Janeiro, sendo avaliado o cumprimento dos procedimentos recomendados. As gestantes de cor preta não tiveram diferença quanto à solicitação de exames, mas a realização foi menor que a das pardas e brancas.11 Portanto, mesmo quando o acesso e a utilização são semelhantes, pode haver desigualdade no processo do pré-natal.

Quanto aos estudos nos quais a cor da pele não mostrou associação com o pré-natal inadequado,14-16 devemos considerar diferentes contextos socioculturais nas regiões brasileiras, diferenças populacionais na distribuição dos fatores de risco e diferenças metodológicas: modelos de análise, opções de ajuste de fatores de confundimento e formas de aferição das variáveis. Deve ser ressaltado que o estudo de Pelotas comparou a cor auto-referida e aquela identificada pelo pesquisador e houve semelhança nos resultados. ${ }^{15}$ Outra hipótese para explicar esta discordância seriam diferentes cenários de discriminação nos serviços de saúde no Brasil.27

Além da discriminação, a variável cor da pele deve ser discutida como proxy de nível socioeconômico e cultural. A desvantagem econômica e social seria um dos mecanismos explicativos das desigualdades raciais de saúde. 18 A correlação entre estas variáveis tem sido observada na área maternoinfantil. Estudos ecológicos nacionais, 28,29 mostram correlação entre poucas consultas de pré-natal, baixa escolaridade, cor da pele preta e residência em regiões de baixa renda. Na cidade do Rio de Janeiro, em estudo transversal, foi observada escolaridade baixa mais frequente em puérperas de cor preta. 10 Portanto, renda e escolaridade devem ser consideradas quando se discutem associações da cor da pele com desfechos de saúde.

Concordando com outros estudos realizados no Brasil,11-13,28-30 a idade da gestante menor ou igual a 19 anos foi um fator predisponente associado ao prénatal inadequado. Além das desigualdades socioeconômicas, que podem explicar em parte esta associação,28,29 questões específicas da gravidez na adolescência, como dificuldade em planejar o futuro, 30 podem contribuir para uma menor adesão ao pré-natal.

A ausência de companheiro, que pode traduzir falta de apoio social e stress emocional durante a gravidez, foi confirmada em nosso estudo como fator para pré-natal inadequado, corroborando os resultados na cidade do Rio de Janeiro11,23 e em outras cidades. 13,14

Entretanto, as mulheres na condição de chefe de família não apresentaram maior frequência de prénatal inadequado, diferindo dos resultados de Bernardes et al.13 Essa discordância pode ser explicada porque estes autores estudaram a base populacional da cidade de São Luís, onde o papel de chefe de família, quando ocupado pela mulher, pode apenas refletir um nível socioeconômico mais baixo, enquanto o estudo atual abrangeu apenas usuárias do SUS, e a detecção desta diferença pode não ter ocorrido pela homogeneidade socioeconômica da população.

Quanto à paridade, os resultados foram semelhantes a outros estudos, ${ }^{11,13}$ que mostram que mulheres na primeira gravidez têm maior chance de realizar pré-natal adequado, assim como as multíparas de realizar pré-natal inadequado. Mulheres com mais filhos podem se considerar experientes e demandar menos a assistência pré-natal ou podem ter experiências negativas de consultas anteriores. ${ }^{11,13}$ Também podem estar mais sobrecarregadas na criação dos filhos e dispor de menos tempo para frequentar serviços de saúde.

Um resultado inesperado foi a ausência de associação com escolaridade, mostrada em vários estudos nacionais e internacionais. $10-13,16,17,28-30 \mathrm{O}$ único trabalho nacional que não identificou escolaridade como fator associado ao pré-natal inadequado 
foi o de Bassani et al., ${ }^{14}$ que também estudou uma população de baixa renda em Porto Alegre. A maioria dos estudos abordou populações completas, sendo mais fácil detectar o contraste. A homogeneidade em relação à baixa escolaridade em populações de baixa renda pode ter dificultado o achado de associações.

Embora sugerisse associação positiva, a variável renda perdeu significância após o ajuste na análise multivariada. Outros estudos têm mostrado esta associação de maneira mais evidente. ${ }^{13-15}$ Vale lembrar que, analogamente à homogeneidade detectada para escolaridade, a população estudada tinha padrão semelhante de renda familiar mensal per capita, com valores muito baixos, cerca de $70 \%$ com menos de um salário-mínimo vigente à época. Nossa hipótese é que esta homogeneidade tenha dificultado evidenciar o contraste e a real associação com a inadequação do pré-natal. A mesma situação foi encontrada em usuárias do SUS no município do Rio de Janeiro. ${ }^{11}$ Além disto, houve ausência de informação desta variável para quase $10 \%$ das participantes, por ignorarem o valor.

$\mathrm{O}$ atendimento pré-natal em serviço público mostrou efeito protetor, diminuindo em 33\% a chance de pré-natal inadequado. Este resultado foi diferente do observado em São Luís, 13 onde o atendimento privado pode estar relacionado ao melhor nível socioeconômico, já que toda base populacional foi estudada. No presente estudo, as mulheres tinham nível socioeconômico mais baixo e, quando relataram uso de serviços particulares, poderiam estar se referindo a atendimento em clínicas de periferia, a preços acessíveis, onde nem sempre o atendimento é qualificado.

Um das limitações do estudo refere-se à amostra, selecionada de forma não aleatória. No entanto, considerando o perfil das maternidades, que constituem referências locais/regionais, além do elevado número de partos realizados, podemos concluir que possivelmente a amostra foi representativa da população de gestantes usuárias do SUS nas regiões metropolitanas abrangidas.

Outros indicadores de qualidade do pré-natal,

\section{Referências}

1. Bhutta ZA, Das JK, Bahl R, Lawn JE, Salam RA, Paul VK, Sankar MJ, Blencowe H, Rizvi A, Chou VB, Walker N, Lancet Newborn Interventions Review Group, Lancet Every Newborn Study Group. Can available interventions end preventable deaths in mothers, newborn babies, and stillbirths, and at what cost? Lancet. 2014; 384 (9940): 347 70 . como a realização de procedimentos clínicos e de exames laboratoriais, não foram abordados nesse estudo, e certamente contribuiriam para a validade do desfecho inadequação do pré-natal. Optamos por adotar o índice de Kotelchuck, largamente empregado em estudos de pré-natal, mas adaptando o número de consultas de acordo com estudos nacionais, considerando que o "número ideal de consultas" é ainda controverso.

Também podemos citar como limitação, o uso de razão de chances como medida de associação, tendo em vista o desenho do estudo, de corte seccional, no qual as informações sobre a exposição e o desfecho são obtidas em um único ponto no tempo. Porém, fomos cautelosos ao interpretar os resultados, informando simplesmente as associações encontradas.

Conclui-se que a cor da pele preta parece se manter como fator relacionado à inadequação na atenção pré-natal. São necessárias políticas de saúde voltadas à redução das iniquidades de raça/cor na assistência pré-natal na população usuária do SUS. Futuras pesquisas podem aprofundar a relação da cor com outras medidas de adequação do pré-natal e ampliar a compreensão dos mecanismos de discriminação no ciclo gravídico-puerperal.

\section{Agradecimentos}

À Direção e ao Centro de Estudos das maternidades nos municípios de Niterói e Rio de Janeiro. À Faculdade de Saúde Pública - USP (FAPESP - edital PPSUS 2009, processo $n^{\circ}$ 09/53253-8) e ao respectivo coordenador da Pesquisa, professor Ruy Laurenti, ao Instituto Fernandes Figueira - FioCruz (CNPq - edital/20/2010, processo $n^{\circ} 402426 / 2010$ 0), Instituto de Estudos em Saúde Coletiva - IESCUFRJ (bolsas - PET Saúde/Vigilância em Saúde, contemplado pelo edital n 07 de 03 de março de 2010 - homologado pela Portaria n 07 de 25/05/2010 -Ministério da Saúde, e bolsas de iniciação científica FAPERJ) e Instituto de Saúde Coletiva - ISC-UFF (bolsas de iniciação científica CNPq e FAPERJ).
2. Kassar SB, Melo AMC, Coutinho SB, Lima MC, Lira PIC. Determinants of neonatal death with emphasis on health care during pregnancy, childbirth and reproductive history. J Pediatr (Rio J). 2013; 89 (3): 269-77.

3. Wehby GL, Murray JC, Castilla EE, Lopez-Camelo JS, Ohsfeldt RL. Prenatal care effectiveness and utilization in Brazil. Health Policy Plan. 2009; 24: 175-88. 
4. Woodhouse C, Lopez Camelo J, Wehby GL. A comparative analysis of prenatal care and fetal growth in eight South American countries. PLoS One. 2014; 9 (3): e91292.

5. Leite RM, Araújo TV, Albuquerque RM, Andrade AR, Duarte Neto PJ. Fatores de risco para mortalidade materna em área urbana do Nordeste do Brasil. Cad Saúde Pública. 2011; 27 (10): 1977-85.

6. Moraes AP, Barreto SM, Passos VM, Golino PS, Costa JE, Vasconcelos MX. Severe maternal morbidity: a casecontrol study in Maranhão, Brazil. Reprod Health. 2013; 10: 11 .

7. Pacheco AJ, Katz L, Souza AS, de Amorim MM. Factors associated with severe maternal morbidity and near miss in the São Francisco Valley, Brazil: a retrospective, cohort study. BMC Pregnancy Childbirth. 2014; 14: 91

8. Viellas EF, Domingues RMSM, Dias MAB, Gama SGN, Filha MMT, Costa JV, Bastos MH, Leal MC. Assistência pré-natal no Brasil. Cad Saúde Pública. 2014; 30: S85-100.

9. Andreucci CB, Cecatti JG. Desempenho de indicadores de processo do Programa de Humanização do Pré-natal e Nascimento no Brasil: uma revisão sistemática. Cad Saúde Pública 2011; 27 (6): 1053-64.

10. Leal MC, Gama SG, Cunha CB. Desigualdades raciais, sociodemográficas e na assistência ao pré-natal e ao parto, 1999-2001. Rev Saúde Pública 2005; 39 (1): 100-7.

11. Domingues RM, Leal Mdo C, Hartz ZM, Dias MA, Vettore MV. Acesso e utilização de serviços de pré-natal na rede SUS do município do Rio de Janeiro, Brasil. Rev Bras Epidemiol. 2013; 16 (4): 953-65

12. Fonseca SC, Monteiro DSA, Pereira CMSC, Scoralick ACD, Jorge MG, Rozario S. Desigualdades no pré-natal em cidade do Sudeste do Brasil. Ciên Saúde Coletiva. 2014; 19 (7): 1999-2010

13. Bernardes AC, Silva RA, Coimbra LC, Alves MT, Queiroz RC, Batista RF, Bettiol H, Barbieri MA, Silva AA Inadequate prenatal care utilization and associated factors in São Luís, Brazil. BMC Pregnancy and Childbirth. 2014; 14 (1): 266.

14. Bassani DG, Surkan PJ, Olinto MT. Inadequate use of prenatal services among Brazilian women: the role of maternal characteristics. Int Perspect Sex Reprod Health. 2009; 35 (1): 15-20.

15. Victora CG, Matijasevich A, Silveira MF, Santos IS, Barros AJD, Barros FC. Socioeconomic and ethnic group inequities in antenatal care quality in the public and private sector in Brazil. Health Policy Plan. 2010; 25 (4): 253-61.

16. Dias-da-Costa JS, Cesar JA, Haag CB, Watte G, Vicenzi K, Schaefer R. Inadequação do pré-natal no Nordeste do Brasil: prevalência e alguns fatores associados. Rev Bras Saúde Matern Infant. 2013; 13 (2): 101-9.

17. Feijen-de Jong EI, Jansen DE, Baarveld F, van der Schans CP, Schellevis FG, Reijneveld SA. Determinants of late and/or inadequate use of prenatal healthcare in high-income countries: a systematic review. Eur J Public Health. 2012; 22 (6): 904-13.

Recebido em 30 de dezembro de 2014

Versão final apresentada em 6 de março de 2015

Aprovado em 2 de abril de 2015
18. Chor D, Lima CR. Aspectos epidemiológicos das desigualdades raciais em saúde no Brasil. Cad Saúde Pública. 2005; 21 (5): 1586-94.

19. Matijasevich A, Victora CG, Barros AJ, Santos IS, Marco PL, Albernaz EP, Barros FC. Widening ethnic disparities in infant mortality in southern Brazil: comparison of 3 birth cohorts. Am J Public Health. 2008; 98 (4): 692-68.

20. Bairros FS, Meneghel SN, Dias-da-Costa JS, Bassani DG, Menezes AM, Gigante DP, Olinto MT. Desigualdades raciais no acesso à saúde da mulher no Sul do Brasil. Cad Saúde Pública. 2011; 27 (12): 2364-72.

21. Laurenti R, Siqueira AAF, Mello-Jorge MHP, Gotlieb SLD, Pimentel EC. Perinatal mortality in hospitals of the state of São Paulo: methodological aspects and some characteristics of mothers and conceptuses. Rev Bras Crescimento Desenvolv Hum. 2013; 23 (3): 261-9.

22. Kotelchuck M. An evaluation of the Kessner adequacy of prenatal care index and a proposed adequacy of prenatal care utilization index. Am J Public Health. 1994, 84: 141420

23. Leal MC, Gama SGN, Ratto KMN, Cunha CB. Uso do índice de Kotelchuck modificado na avaliação da assistência pré-natal e sua relação com as características maternas e o peso do recém-nascido no Município do Rio de Janeiro. Cad Saúde Pública. 2004; 20 (Supl. 1): 63-72.

24. Andersen RM. Revisiting the behavioral model and access to medical care: does it matter? J Health Soc Behav. 1995; 36: $1-10$.

25. Victora CG, Huttly SR, Fuchs SC, Olinto MT. The role of conceptual frameworks in epidemiological analysis: a hierarchical approach. Int J Epidemiol. 1997; 26 (1): 224-7.

26. Moubarac J. Persisting problems related to race and ethnicity in public health and epidemiology research. Rev Saúde Pública. 2013; 47 (1): 105-16.

27. Bailey SR, Loveman M, Muniz JO. Measures of "Race" and the analysis of racial inequality in Brazil. Soc Sci Res. 2013; 42 (1): 106-19.

28. Friche AA, Caiaffa WT, César CC, Goulart LM, Almeida MC. Indicadores de saúde materno infantil em Belo Horizonte, Minas Gerais, Brasil, 2001: análise dos diferenciais intra-urbanos. Cad Saúde Pública. 2006; 22 (9): 195565

29. Predebon KM, Mathias TA, Aidar T, Rodrigues AL. Desigualdade sócio-espacial expressa por indicadores do Sistema de Informações sobre Nascidos Vivos (SINASC). Cad Saúde Pública. 2010; 26 (8): 1583-94.

30. Gama SGN, Szwarcwald CL, Sabroza AR, Branco VC, Leal MC. Fatores associados à assistência pré-natal precária em uma amostra de puérperas adolescentes em maternidades do município do Rio de Janeiro, 1999-2000. Cad Saúde Pública. 2004; 20 (Supl. 1): 101-11. 